\title{
A Pilot Study of Problem Gambling among Student Online Gamblers: Mood States as Predictors of Problematic Behavior
}

\author{
Neil Matthews, B.Sc., Bill Farnsworth, M.Phil., and Mark D. Griffiths, Ph.D.
}

\begin{abstract}
Within the last decade, interest in online gambling has increased. This pilot study examined online gambling among students to identify the extent to which student Internet gamblers manifest a propensity for problem gambling and to understand if mood states at various times are predictors of problem gambling. A questionnaire was administered to 127 student Internet gamblers. In addition to questions asking for basic demographic data, the questionnaire included the Positive and Negative Affect Schedule (PANAS) and the South Oaks Gambling Screen (SOGS). Results showed that approximately one in five online gamblers (19\%) was defined as a probable pathological gambler using the SOGS. Among this sample, results also showed that problem gambling was best predicted by negative mood states after gambling online and negative mood states more generally.
\end{abstract}

\section{Introduction}

$\mathbf{T}$ HE INTRODUCTION OF INTERNET GAMBLING has caused much concern and debate because it is believed that the Internet may facilitate problematic gambling behavior. ${ }^{1}$ As a result of the almost exponential growth of the Internet along with people's desire to gamble, an increasing number of online gambling and betting opportunities are available. In 1997, approximately 30 gambling Web sites were available. ${ }^{2}$ By 2007, an estimated 3,000 gambling Web sites existed on the Internet. ${ }^{3}$ According to the latest British Gambling Prevalence Survey, ${ }^{4}$ overall only a small proportion of the population (6\%) had gambled via the Internet in the previous year, although this was a significant increase from the previous survey in 1999 that showed less than $1 \%$ had gambled on the Internet. ${ }^{5}$ Although relatively small, the increase in online gambling participation is clearly evident. Furthermore, the most recent British Gambling Prevalence Survey found that the rate of problem gambling was 5\% among Internet gamblers but only $0.5 \%$ among those who had never accessed the Internet to gamble. ${ }^{6}$

Empirical research into online problem gambling is relatively limited. Evidence to date suggests a relationship between Internet gambling and problem gambling, but it is far from conclusive. However, a small number of self-selected sample studies suggest there is a relationship. ${ }^{7-9}$ Research by Wood and Williams ${ }^{9}$ on a self-selected sample of Internet gamblers $(n=1,920)$ in North America highlighted a strong relationship between Internet gambling and problem gambling with $43 \%$ of the sample meeting the criteria for either moderate or severe problem gambling. Another survey carried out on a self-selected sample in the United States also found that Internet gamblers were more likely than nonInternet gamblers to be problem gamblers. ${ }^{8}$

Griffiths and Barnes ${ }^{7}$ examined differences between a selfselected sample of 473 student Internet gamblers and nonInternet gamblers. Internet gamblers were more likely to be male than female, more likely to be problem gamblers than non-Internet gamblers, and males were significantly more likely than females to be problem Internet gamblers. They suggested the structural and situational characteristics of Internet gambling may have a negative psychosocial impact on Internet gambling. Other research has focused on specific online gambling activities and problem gambling. For example, Wood et al. ${ }^{10}$ studied student online poker players and reported that nearly one in five of their sample (18\%) were problem gamblers according to DSM-IV criteria. ${ }^{11}$

The findings from Wood et al. ${ }^{10}$ demonstrated that problem gambling among student online poker players was best predicted by negative mood states after playing and by playing to escape from problems. Negative mood states were experienced as a consequence of gambling, which in turn facilitated the need for many of the gamblers to modify their mood through playing again. In addition, heart rate measurement studies suggest gambling is an exciting behavior, and some form of arousal is vital in reinforcing that

Psychology Division, Nottingham Trent University, Nottingham, United Kingdom. 
behavior. ${ }^{12}$ The pathway model of problem gambling theorizes that gamblers who are emotionally unstable use gambling to alter mood states and/or to meet psychological desire. ${ }^{13}$ Evidence from numerous studies, focusing on different gambling activities, shows that both mood states and escapism reinforce gambling behavior. ${ }^{14,15}$ Research also suggests a close link between problem gambling and mood disorders such as manic depression. ${ }^{16}$ The aim of the current study was to identify to what extent student Internet gamblers manifest a propensity for problem gambling, in order to extend research on the relationships among online gambling, problem gambling, and mood states. Based on past literature, it was hypothesized that (a) a high negative mood state would significantly predict problem gambling, (b) a high positive mood state while gambling online would significantly predict problem gambling, and (c) a high negative mood state directly after gambling online would significantly predict problem gambling.

\section{Method}

\section{Participants}

A total of 127 participants took part in the study ( 86 males, 41 females). All participants were self-defined online gamblers who had participated in at least one online gambling experience in their lifetime. Their average age was 20.8 years $(S D=1.9$ years). Participants were mostly recruited from a university in the Midlands area of the United Kingdom. This particular university is representative of a wide geographical location because it attracts students from a large number of cities across the United Kingdom.

\section{Design and materials}

A questionnaire with 100 closed questions was constructed that included three main sections: questions concerning demographic information, questions from the South Oaks Gambling Screen (SOGS), and questions from the Positive and Negative Affect Schedule (PANAS).

Demographics. Questions were asked relating to sex, age, and frequency of online gambling with participants indicating how often they gamble online on average (i.e., less than once a week, once a week, 2 to 5 times a week, and every day).

South Oaks Gambling Screen (SOGS). The SOGS was developed by Lesieur and Blume ${ }^{17}$ and contains 20 questions on gambling behavior (e.g., Did you ever gamble more than you intended? Have people criticized your gambling? Have you ever felt guilty about the way you gamble or what happens when you gamble?). Those scoring less than 3 on the SOGS are operationally defined as non-problem gamblers, while those scoring 3 to 4 are defined as a problem gamblers and those scoring 5 or more are defined as a probable pathological gamblers.

Positive and Negative Affect Schedule (PANAS). The PANAS, was developed by Watson et al., ${ }^{18}$ contains 10 adjectives describing negative moods (distressed, upset, guilty, scared, hostile, irritable, ashamed, nervous, jittery, afraid) and 10 adjectives describing positive moods (interested, ex- cited, strong, enthusiastic, proud, alert, inspired, determined, attentive, active). Participants indicate to what extent each word describes how they feel at a certain point in time by using a 5-point Likert scale (from very slightly or not at all to extremely). Mean scores on each scale of the PANAS can range from 10 to 50. The psychometric properties of the PANAS have been investigated. ${ }^{18}$ Both subscales show satisfactory internal consistency on large student samples. Internal consistency measuring mood states over different time frames, ranging from at the moment to in general always exceeded 0.84 . Test-retest reliability for the negative mood states and the positive mood states subscales were 0.69 and 0.72 respectively. Validity has been demonstrated by the independence of the two subscales, $r=-0.09$. In this study, the PANAS was used to measure participants' mood states at three different stages: how they felt (a) generally, (b) while gambling online, and (c) directly after gambling on the Internet.

\section{Procedure}

Approximately 3,000 students at universities in the Midlands area of the United Kingdom were asked, via e-mail, to participate in a study on Internet gambling. The latest British Gambling Prevalence Survey showed that $6 \%$ of the population had gambled online in the past year, so it was estimated that of the students who had been contacted, approximately 180 would have had gambled online. Given that 127 participants responded, it was considered that the online sample was fairly representative. Online gamblers had a choice of filling out the questionnaire online or offline. Data were collected over a period of 8 weeks, and all respondents were assured of both confidentiality and anonymity. Ethics approval for the study was granted by the researchers' university Ethics Committee.

\section{Data analysis}

The research aims were addressed by three separate statistical analyses: (a) the association between the type of gambler and the frequency of gambling was investigated by tabulating the frequency of gambling against the type of gambler as defined by the SOGS scores; (b) the association between problem gambling behavior and frequency of gambling behavior was investigated by means of a linear regression with problem gambling behavior as the dependent variable and frequency of online gambling as the predictor variable; (c) the relationship between problem gambling behavior and mood states was investigated by means of a standard multiple regression with the SOGS score for problem gambling as the dependent variable and the six PANAS mood state measures as the predictor variables. These predictor variables were general negative mood state, negative mood state while gambling online, negative mood state after gambling online, general positive mood state, positive mood state while gambling online, and positive mood state after gambling online.

\section{Results}

Results indicated that $19 \%$ of the sample $(n=24)$ were classified as probable pathological gamblers. A further $18 \%$ $(n=23)$ were classified as potential pathological gamblers, and $63 \%(n=80)$ were defined as non-problem gamblers. 
Table 1. Association between Frequency of Online Gambling and Type of Gambler

\begin{tabular}{lccrr}
\hline & Less than once a week & Once a week & 2 to 5 times a week & Every day \\
\hline Non-problem gamblers $(n=80)$ & $71 \%$ & $21 \%$ & $5 \%$ & $3 \%$ \\
Potential pathological gamblers $(n=23)$ & $61 \%$ & $17.5 \%$ & $17.5 \%$ & $4 \%$ \\
Probable pathological gamblers $(n=24)$ & $25 \%$ & $21 \%$ & $42 \%$ & $12 \%$ \\
\hline
\end{tabular}

Although $63 \%$ of respondents were non-problem gamblers, $41 \%(n=52)$ endorsed one or two of the SOGS responses affirmatively (see Table 1).

Table 1 shows that as the severity of the problem gambling classification increases, the frequency of gambling also increases, particularly in the "2 to 5 times a week" and "daily" categories. Because no control group was used in this study, one-sample $t$ tests were conducted on the mean scores of each of the mood state measures comparing them against the scale norm of 30. They were all significantly different except for positive affect while gambling. The mean scores for the respondents' general mood states were 19 (of 50) $(t=-16.68$, $d f=126 ; p<0.001$ ) on the negative affect scale and 31 (of 50) $(t=2.19, d f=126 ; p<0.05)$ on the positive affect scale. The mean scores for the respondents' mood states while gambling online were 21 (of 50) $(t=-13.84, d f=126 ; p<0.001)$ on the negative affect scale and 29 (of 50$)(t=-0.74, d f=126$; $p>0.05)$ on the positive affect scale. The mean scores for the respondents' mood states after gambling online were 21 (of 50) $(t=-12.17, d f=126 ; p<0.001)$ on the negative affect scale and 22 (of 50) $(t=-12.33, d f=126 ; p<0.001)$ on the positive affect scale.

The mean scores of each mood state variable over the whole sample corresponded well with previously tested samples consisting of undergraduates enrolled in various psychology courses ${ }^{18}$ (i.e., general negative mood state mean $=18.6, S D=7.7$; positive mood state while gambling online mean $=29.4, S D=8.7$; negative mood state directly after gambling online mean $=20.6, S D=8.7$ ). Therefore, the data on mood state collected from the present study appear fairly representative of other populations. Additionally, the mean scores showed that the overall sample reported more positive affects during gambling $(M=29.4)$ compared to less positive affects directly after gambling online $(M=21.9)$. An independent samples t-test was carried out to see if there was statistically significant difference in mean values between positive mood states during and directly after gambling online. Results showed that there was a statistically significant difference between the means of the two conditions $(t=7.48$, $d f=252 ; p<0.05)$.

A linear regression was performed to see if there was an association between problem gambling behavior and frequency of online gambling. The regression was a good fit (adjusted $R^{2}=0.14$ ), indicating that $14 \%$ of the model can be generalized to other populations. The variable of online gambling frequency proved to be significant $[F(1,126)=21.6$, $p<0.01]$. The standardized regression coefficient also showed that online gambling frequency was a good predictor of problem gambling behavior $(\beta=0.383)$. As expected, there was a significant positive relationship between the severity of gambling behavior and the frequency of online gambling. As the frequency of gambling increased, the number of gambling-related problems increased.
Colinearity between the six independent measures of mood states was assessed as part of the multiple regression. Colinearity exists when a regression model has two or more predictors with a strong correlation. If colinearity exists between two variables within regression analysis, it becomes difficult to estimate their individual regression coefficients reliably. The output for these diagnostic tests included (a) the variance inflation factor (VIF; if the VIF is greater than ' 10 , there is cause for concern, and if average VIF is far greater than 1, regression may be biased); (b) the tolerance value (TV; a TV below 0.1 indicates a serious problem; a TV below 0.2 indicates a potential problem); and (c) condition index (CI; a $\mathrm{CI}$ greater than 30 is considered indicative of colinearity). ${ }^{19}$ Within the current regression model, the VIF values were all below 10 (1.96-5.07), and the tolerance values ranged from 0.197 to 0.65 . Five of them were above 0.2 (ranging from 0.299 to 0.649 ), and the one at 0.197 is very close to the cutoff value. Additionally, the largest CI was 21.83 (below the cutoff condition index of 30 for colinearity). These results suggest there is no colinearity within the data and the values for each variable are not interchangeable. Thus, it is possible to obtain unique estimates of the regression coefficients.

The analysis of variance (ANOVA) tests whether the regression model is significantly better at predicting the outcome than is making a best-guess prediction using the mean. The regression model in this study was significant, as indicated by the ANOVA results $(p<0.001)$. Two of the six variables that were measured also proved to be significant $[F(6$, $127)=17.54, p<0.001]$. Thus, the regression model significantly improves the ability to predict the outcome variable. The general regression model was a good fit (adjusted $R^{2}=0.441$ ), indicating that $44 \%$ of the model can be generalized to other populations. The difference between the value of $R^{2}$ and the adjusted $R^{2}$ for this model was small, $0.47-0.44=0.03$, or about $3 \%$. This indicates that if the model were derived from the general population instead of this sample, it would account for approximately 3\% less variance in the outcome. Consequently, the small difference between the $R$ values also suggests the model generalizes well. The significant variables that predicted problem gambling, as measured by SOGS scores, were negative mood states in general and negative mood states directly after gambling online. The standardized regression coefficients showed that negative mood state after gambling online was the best predictor of problem gambling $(\beta=0.617)$, and negative mood state more generally was the next best predictor $(\beta=0.2)$. The other four SOGS scores did not significantly predict problem gambling behavior (see Table 2).

\section{Discussion}

This study attempted to identify the extent to which student Internet gamblers manifest a propensity for problem 
Table 2. Predictors of Problem Gambling among ONLINE GAMBLERS

\begin{tabular}{lccc}
\hline Variable & $\beta$ value & t value & $p$ value \\
\hline $\begin{array}{l}\text { General negative } \\
\text { mood state }\end{array}$ & 0.200 & 2.14 & $\mathbf{0 . 0 3 4}$ \\
$\begin{array}{l}\text { Negative mood } \\
\quad \text { state while gambling }\end{array}$ & -0.185 & -1.52 & 0.131 \\
$\begin{array}{l}\text { Negative mood state } \\
\quad \text { after gambling }\end{array}$ & 0.617 & 4.11 & $\mathbf{0 . 0 0 1 *}$ \\
$\begin{array}{l}\text { General positive } \\
\text { mood state }\end{array}$ & 0.085 & 1.03 & 0.305 \\
$\begin{array}{l}\text { Positive mood state } \\
\quad \text { while gambling online }\end{array}$ & 0.137 & 1.28 & 0.312 \\
$\begin{array}{l}\text { Positive mood state } \\
\text { after gambling }\end{array}$ & -0.141 & -1.45 & 0.148 \\
\hline
\end{tabular}

$* p<0.05$.

gambling and to understand if mood states at various times are predictors of problem gambling. Perhaps the most salient finding was that over one third of the sample (37\%) was classified with having some sort of gambling problem (i.e., potential pathological gambler, $18 \%$; probable pathological gambler, 19\%). The frequency of online gambling among the groups of gamblers suggested a strong association between online gambling and problem gambling. This was emphasized by the fact that $75 \%$ of probable pathological gamblers compared with only $29 \%$ of non-problem gamblers gambled online at least once a week on average. Furthermore, 54\% of probable pathological gamblers gambled online at least twice a week, giving further support for the link between online gambling and problem gambling. When considering rates of problem gambling among this sample compared to rates among the general population, evidence suggests that Internet gamblers are more likely than non-Internet gamblers to develop problems. As well as relating to UK student-based samples, the findings presented here are similar to research carried out in other countries. The prevalence of moderate to severe problem gambling in a Canadian adult population of Internet gamblers was $43 \%{ }^{9}$ compared to $37 \%$ in this study (although it must be noted that both studies used self-selected samples).

The results indicate that students may indeed be vulnerable to problem gambling on the Internet. With nearly $40 \%$ of the sample gambling online at least once a week, this study appears to confirm previous research on student online gambling ${ }^{7,10,20}$ that Internet gambling is a popular form of gambling among some students. It could perhaps be argued that the Internet may be facilitating problems that were previously nonexistent and/or may be providing a highly accessible and convenient medium for those who are already predisposed to problem gambling. In relation to mood states, the best predictor for problem gambling among this sample was the feeling of negative mood states directly after gambling online. Another predictor was high scoring on the negative affect scale more generally. The results from this study suggest that problem Internet gamblers are people who generally feel a broad range of negative mood states. These moods (as measured by the PANAS) include anger, disgust, scorn, guilt, fearfulness, depression, and so on. Overall, respondents reported feeling more positive than negative while gambling online. This suggests that while gambling online, students generally feel fairly energetic, excited, and enthusiastic. However, feeling in a positive mood state while gambling online was not a significant predictor of problem gambling.

The negative impact that Internet gambling can have on people's mood states is supported by the differences in the mean scores between the predictor variables. The mean score for the respondents' general negative mood state was lower than for negative mood state after gambling online. This suggests that gambling online has an increased negative affect on a student gambler's mood state. Participants also reported feeling more positive when they gambled online compared to positive mood state more generally, although they reported feeling less positive overall directly after online gambling when compared to positive mood more generally. It appears that gambling online creates positive moods in the short term (i.e., while gambling), which suggests that those who experienced greater negative feelings before and after gambling online were more likely to gamble again in order to modify their mood state. This suggests that gambling is being used as a response to block out negative mood states and supports theories that hypothesize problem gambling as an escapebased coping strategy ${ }^{15}$ while also providing empirical support for the pathway model of problem gambling. ${ }^{13}$

The study also supports the findings from Wood et al., ${ }^{10}$ who found that negative mood states after gambling were a strong predictor of problem gambling among their sample of student online poker players. Most research on the relationship between mood states and gambling has concentrated on nonremote forms, such as slot machine gambling. ${ }^{14}$ Also, findings from poker machine players suggest that persistence is accounted for by prior mood. ${ }^{21}$ The results also give some support for several other studies that relate mood disorders with problem gambling. ${ }^{16,22}$

There are a number of limitations to this study. The sample was self-selected and as a result is not representative of the general population. Also, the study examined all forms of Internet gambling and did not differentiate between different gambling types. This may be considered a limitation because various online gambling activities have different characteristics. For example, online poker is very different from online bingo, which is very different from online lotteries. More research is needed to compare problem gambling rates across specific online gambling activities. Another possible limitation concerns the scale used to measure problem gambling. This study used the SOGS, which may overinflate the rate of problem gambling. ${ }^{4,23,24}$ However, a recent review of the literature concluded that the SOGS scale was valid. ${ }^{25}$ Finally, the study used a relatively small number of participants, although the fact that such significant differences were found in a relatively modest population suggests the significant differences are robust.

\section{Disclosure Statement}

No competing financial interests exist.

\section{References}

1. Griffiths MD. (2007) Gambling addiction and its treatment within the NHS. London: British Medical Association, pp. $1-44$. 
2. Azmier JJ. (2001) Gambling @ home: Internet gambling in Canada. www.gamblinglicenses.com/PDF/canada_internet_ gambling.pdf (accessed January 5, 2009).

3. Gamcare. (2007) Online gambling. www.gamcare.org.uk/ pages/online.html (accessed April 19, 2008).

4. Wardle H, Sproston K, Orford J, et al. (2007) British Gambling Prevalence Survey 2007. London: National Centre for Social Research

5. Sproston K, Erens B, Orford J. (1999) Gambling behaviour in Britain: results from the British Gambling Prevalence Survey. London: National Centre for Social Research.

6. Griffiths MD, Wardle J, Orford J, et al. Socio-demographic correlates of Internet gambling: findings from the 2007 British Gambling Prevalence Survey. CyberPsychology \& Behavior 2009; 12:199-202.

7. Griffiths MD, Barnes A. Internet gambling: an online empirical study among gamblers. International Journal of Mental Health Addiction 2008; 6:194-204.

8. Ladd GT, Petry NM. Disordered gambling among university-based medical and dental patients: a focus on Internet gambling. Psychology of Addictive Behaviours 2002; 16: 76-9.

9. Wood RTA, Williams RJ. Problem gambling on the Internet: implications for Internet gambling policy in North America. New Media \& Society 2007; 9:520-42.

10. Wood RTA, Griffiths MD, Parke J. Acquisition, development, and maintenance of online poker playing in a student sample. CyberPsychology \& Behavior 2007; 10:354-61.

11. American Psychiatric Association. (1994) Diagnostic and statistical manual of mental disorders. 4th ed. Washington DC: American Psychiatric Association.

12. Griffiths MD. Tolerance in gambling: an objective measure using the psychophysiological analysis of male fruit machine gamblers. Addictive Behaviors 1993; 18:365-72.

13. Blaszczynski AP, Nower L. A pathways model of problem gambling. Addiction 2002; 97:487-99.

14. Griffiths MD. The role of subjective mood states in the maintenance of fruit machine gambling behaviour. Journal of Gambling Studies 1995; 11:123-35.

15. Wood RTA, Griffiths MD. A qualitative investigation of problem gambling as an escape-based coping strategy. Psychology \& Psychotherapy: Theory, Research \& Practise 2007; 80:107-25.

16. Kim SW, Grant JE, Eckert ED, et al. Pathological gambling and mood disorders: clinical associations and treat- ment implications. Journal of Affective Disorders 2006; 92:109-16.

17. Lesieur HR, Blume SB. The South Oaks Gambling Screen (SOGS): a new instrument for the identification of pathological gamblers. American Journal of Psychiatry 1987; 144:1184-8.

18. Watson D, Clark LA, Tellegen A. Development and validation of brief measures of positive and negative affect: the PANAS scales. Journal of Personality \& Social Psychology 1988; 54:1063-70.

19. Field A. (2000) Discovering statistics using SPSS for Windows. Thousand Oaks, CA: Sage.

20. Griffiths MD, Parke J, Wood RTA, et al. Online poker gambling in university students: further findings from an online survey. International Journal of Mental Health \& Addiction 2009; in press.

21. Dickerson M, Cunningham R, Legg-England S, et al. On the determinants of persistent gambling. III, Personality, prior mood, and poker machine play. International Journal of the Addictions 1991; 26:531-48.

22. Ohtsuka K, Bruton E, DeLuca L. Sex differences in pathological gambling using gaming machines. Psychological Reports 1997; 80:1051-7.

23. Battersby MW, Thomas LJ, Tolchard B, et al. The South Oaks Gambling Screen: a review with reference to Australian use. Journal of Gambling Studies 2002; 18:257-71.

24. Volberg RA. Research methods in the epidemiology of pathological gambling: development of the field and directions for the future. Annuário de Psicologia 1999; 30:33-46.

25. Gambino B, Lesieur H. (2006) The South Oaks Gambling Screen (SOGS): a rebuttal to critics. Journal of Gambling Issues. www.camh.net/egambling/issue17/pdfs/gambino.pdf (accessed January 5, 2009).

Address correspondence to: Dr. Mark D. Griffiths

Professor of Gambling Studies International Gaming Research Unit Psychology Division, Department of Social Sciences Nottingham Trent University Burton Street, Nottingham, NG1 4BU United Kingdom

E-mail: mark.griffiths@ntu.ac.uk. 
